\section{Pushing the Envelope in Atomic Force Microscopy}

\section{Monteith G. Heaton and Jason P. Cleveland Asylum Research, Santa Barbara, CA monte@asylumresearch.com}

Over the past decade, Atomic Force/Scanning Probe Microscopy (AFM/SPM) has emerged as the leading tool for investigations at the nanoscale - doing everything from imaging, to compositional differentiation, to explorations of molecular forces. However, aside from some interesting tweaks, add-ons and repackaging, the field has seen no fundamentally new instruments for several years. For the extremely high-resolution AFM/SPMs, there has literally been no completely new microscope for well over a decade. We report here on the new Cypher ${ }^{\mathrm{TM}}$ AFM from Asylum Research (Figure 1). that delivers upgrades from the existing field of older AFM/SPMs on a variety of levels.

\section{Highest Resolution}

Three-dimensional imaging and resolution is why AFM/SPM has become such a dominant tool in nanoscience and nanotechnology (Figures 2, 3, 4). While Cypher indeed exceeds the open-loop resolution of other commercially-available AFM/SPMs (Figure 5), it is in its closed loop resolution that Cypher truly differentiates itself (Figure 5 inset). A bit of background: almost all commercial AFMs use piezo-electric crystals (piezos, for short) as the transducers that generate the fine scale motion used in scanning the probe across the surface. Piezos are crystals that expand very slightly (typically one part in a thousand) when a voltage is applied to them. Amazingly, these devices can generate motion that is a small fraction of

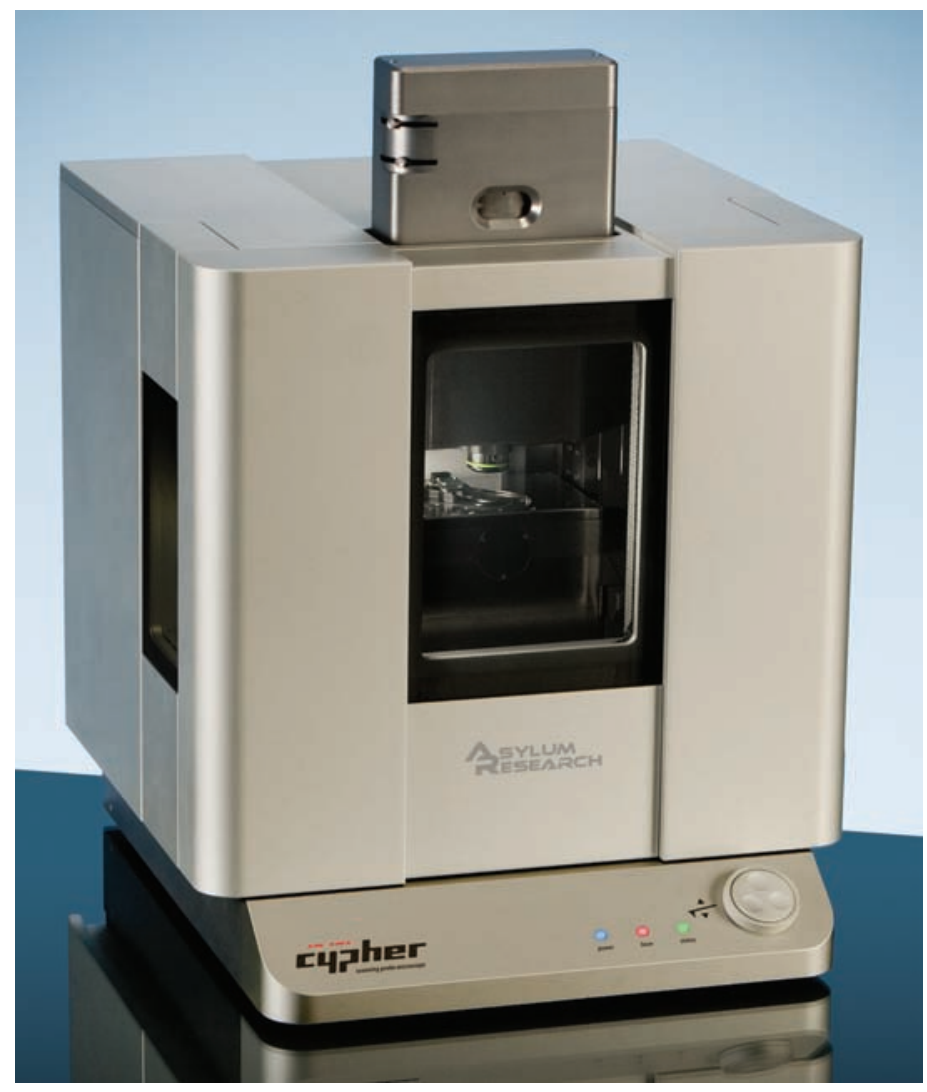

Figure 1: The new Cypher AFM from Asylum Research is the first new small sample AFM in over a decade and offers the highest resolution open loop and closed loop resolution available. the size of a single atom. Their downside is that at larger scan sizes they exhibit non-linearity (their expansion is no longer proportional to voltage) and hysteresis that prevent accurate control of the probe position.

The differences between "Open Loop" and "Closed Loop" systems involve how these piezos are controlled. In simple terms, "Open Loop" refers to operation of the AFM/SPM without positional feedback to compensate for inaccuracies and distortions caused by the piezos in the scanning mechanism. Open loop images can be striking and reveal a great deal of information, but the actual dimensions of features will carry those inaccuracies. Further, open loop systems cannot accurately return the probe to a specific point in an image, so making measurements (e.g. stiffness) at specific points of interest in the image is not possible. In "Closed Loop," the probe position is constantly monitored by an additional sensor and the voltage to the piezos is corrected to keep the probe at its desired position. The result is that the images are more accurate and reflect the true dimensions of the sample features being scanned. The probe can also be precisely returned to points in the image after acquisition, opening a multitude of measurement possibilities.

In the past several years, because of the advantages mentioned above, "Closed Loop" has become a standard feature on many commercial AFMs, but has always come with a major tradeoff. In closed loop operation, the system is using data from the sensors to correct the probe position, so any noise in the sensors directly affects the probe position. Even with sensors with sub-nanometer noise levels, this still means that sensor noise is a significant issue for scan sizes below a few hundred nanometers. Typically, users are forced to switch to open loop operation at these small scan sizes to avoid this noise issue. By pushing sensor noise levels well below the one Angstrom $(0.1 \mathrm{~nm})$ level, the Cypher AFM has effectively eliminated this issue. The Cypher system uses Asylum's patented sensor technology, which is capable of atomic resolution in all three axes. With positioning accuracies better than 60 picometers (0.06 nanometers) in $X, Y$ and $Z$, these Nanopositioning Sensors $\left(\mathrm{NPS}^{\mathrm{TM}}\right.$ ) are the quietest available on an AFM today by a factor of 5 to $10 \times$. Thus, users get the best of both worlds, no longer having to choose between the accuracy and control of closed loop and the low noise of open loop. The result is that Cypher produces the most accurate images, measurements, positioning, and nanomanipulation possible - all at atomic resolution.

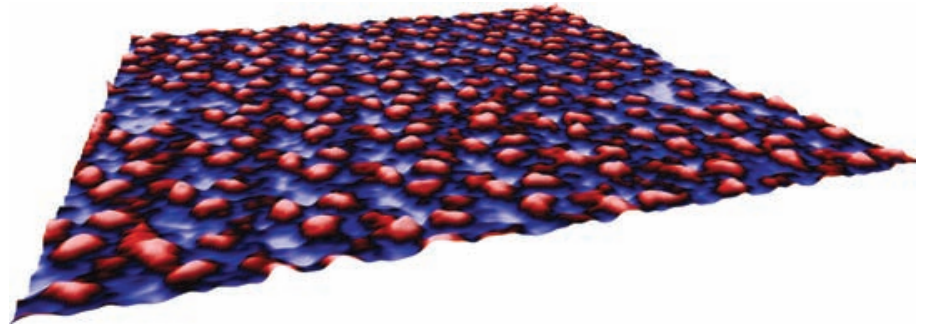

Figure 2: Closed Loop image of extracellular face of bacteriorhodopsin in buffer, 50nm scan.

\section{High Immunity to Vibration and Drift}

To further improve image and measurement resolution and accuracy, Cypher's system-level mechanical design is inherently more immune to normal environmental vibration. The reason is simple: this design includes an extremely short and rigid mechanical path from the sample to the scanning probe - the shorter 


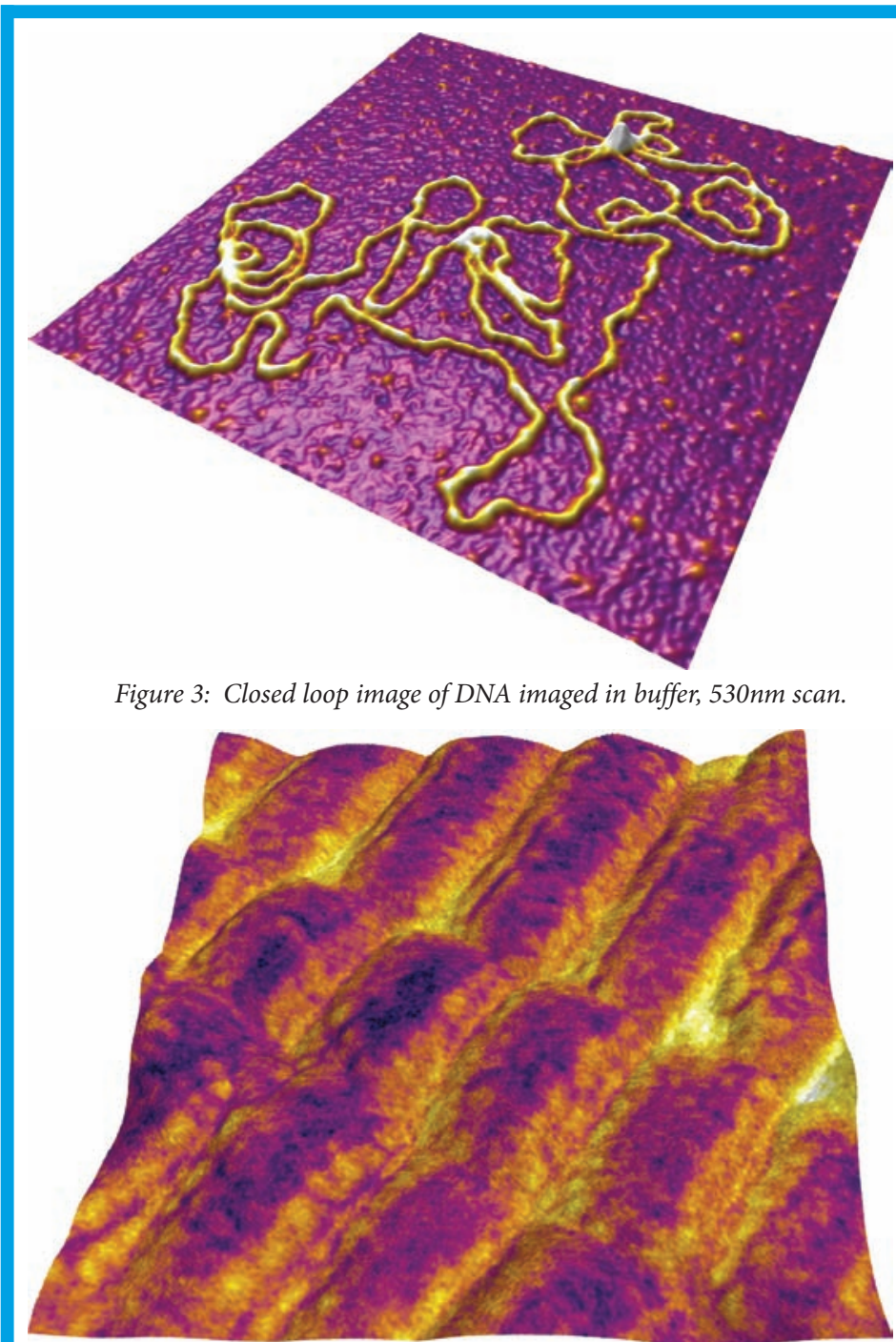

Figure 4: Closed loop DualAC Mode image of collagen. Second mode amplitude is overlaid on topography, 300nm scan.

this mechanical path, the less susceptible the AFM/SPM is to the introduction of noise and thermal drift. The Cypher design is also integrated with additional features to reduce noise and drift, including temperature control within the integrated system enclosure, which allows experiments to be conducted from minutes to hours with minimal thermal drift of the probe position relative to the sample. The enclosure (Figure 1) also provides additional shielding from acoustic noise such that, for most lab environments, Cypher requires no additional vibration or acoustic noise protection, while still routinely providing atomic resolution.

\section{Ease of Use}

In addition to its superior resolution and accuracy, Cypher is also easy to use. Specifically, the process of probe alignment with the laser and photodetector is simplified to a single mouse-click (Figure 6). Cypher also incorporates diffraction-limited optics for sub-micron resolution sample and tip viewing with Kohler illumination to improve operability and ease of use further.

\section{Sub-pico Newton Force Measurements}

One of the most important non-imaging uses of AFMs is the measurement of molecular forces. These measurements are often made in liquid and have contributed greatly to the understanding of forces in molecular biology. For example, the AFM can mea-

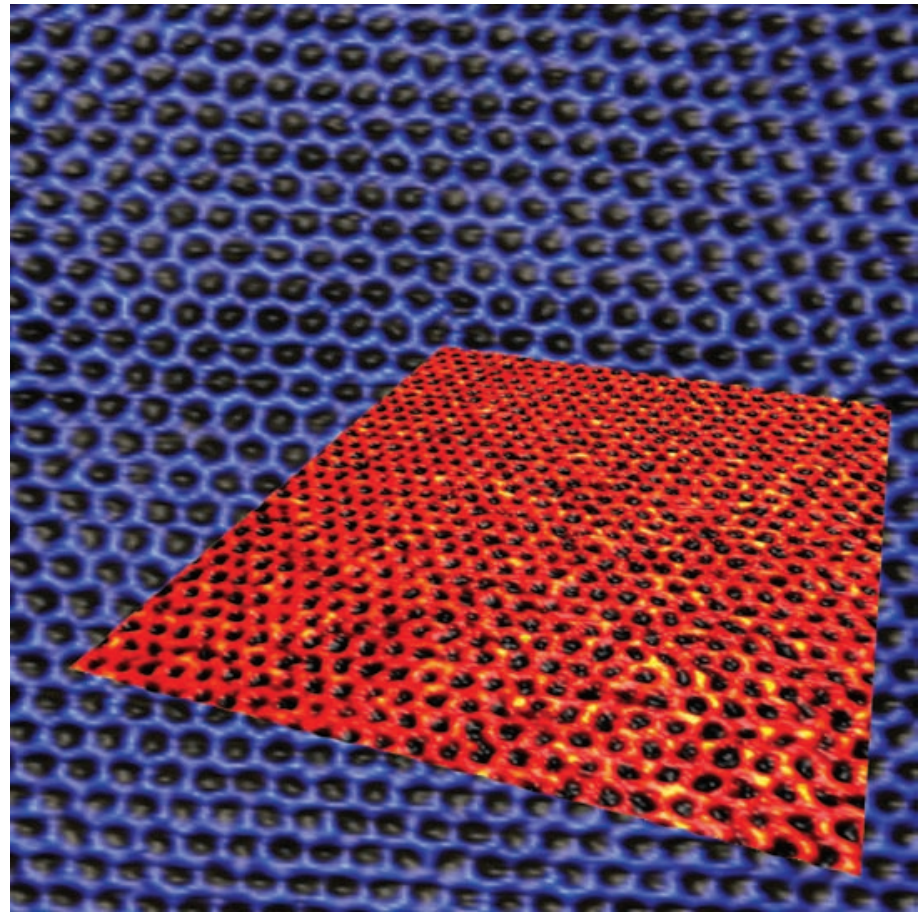

Figure 5: Open loop atomic resolution STM on the cleavage plane of Highly Oriented Pyrolitic Graphite (HOPG), $5 \mathrm{~nm}$ scan. Inset: Closed loop atomic resolution STM on HOPG, $6 \mathrm{~nm}$ scan.

sure the forces required to mechanically unfold a single protein molecule tethered between the tip of the probe and a surface. In liquids, the ultimate limits on force sensitivity are set by thermal noise. Water molecules continually bumping into the cantilever cause a background vibration that can mask extremely small forces. Smaller cantilevers fundamentally reduce this background noise and improve the force sensitivity of the AFM. With its compatibility with extremely small cantilevers, the Cypher AFM pushes the force noise floor into the sub-pico Newton range, achieving a sensitivity heretofore unique to optical tweezers. However, Cypher achieves this force sensitivity at much higher spring constants and with much sharper probes than optical tweezers, allowing measurements to be made in the higher force gradients found near surfaces where the optical tweezer probe would become stuck to the surface.
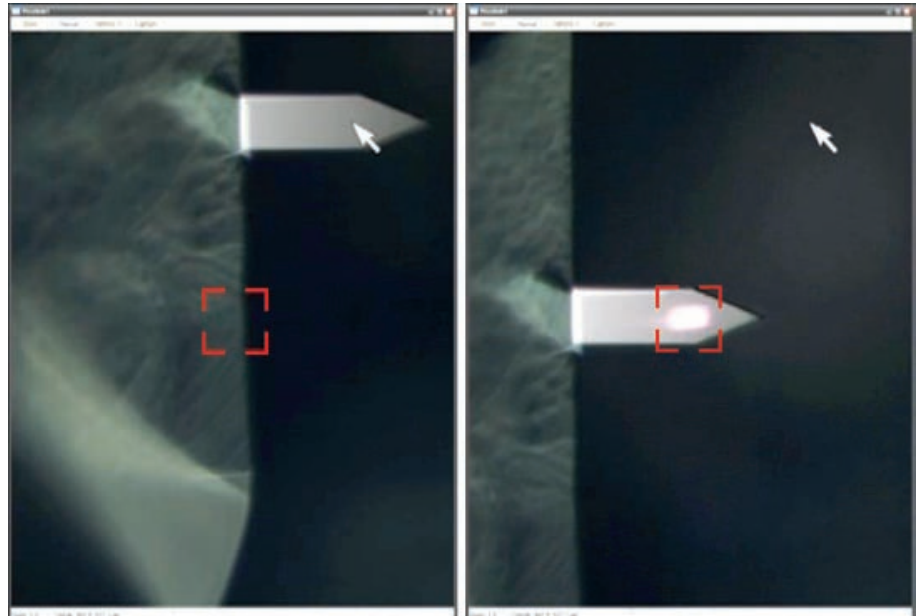

Figure 6: With the Cypher AFM's SpotOn ${ }^{\text {tw }}$ automated laser alignment, the user simply clicks on the desired laser spot location and motorized stages automatically align the laser and photodetector. 


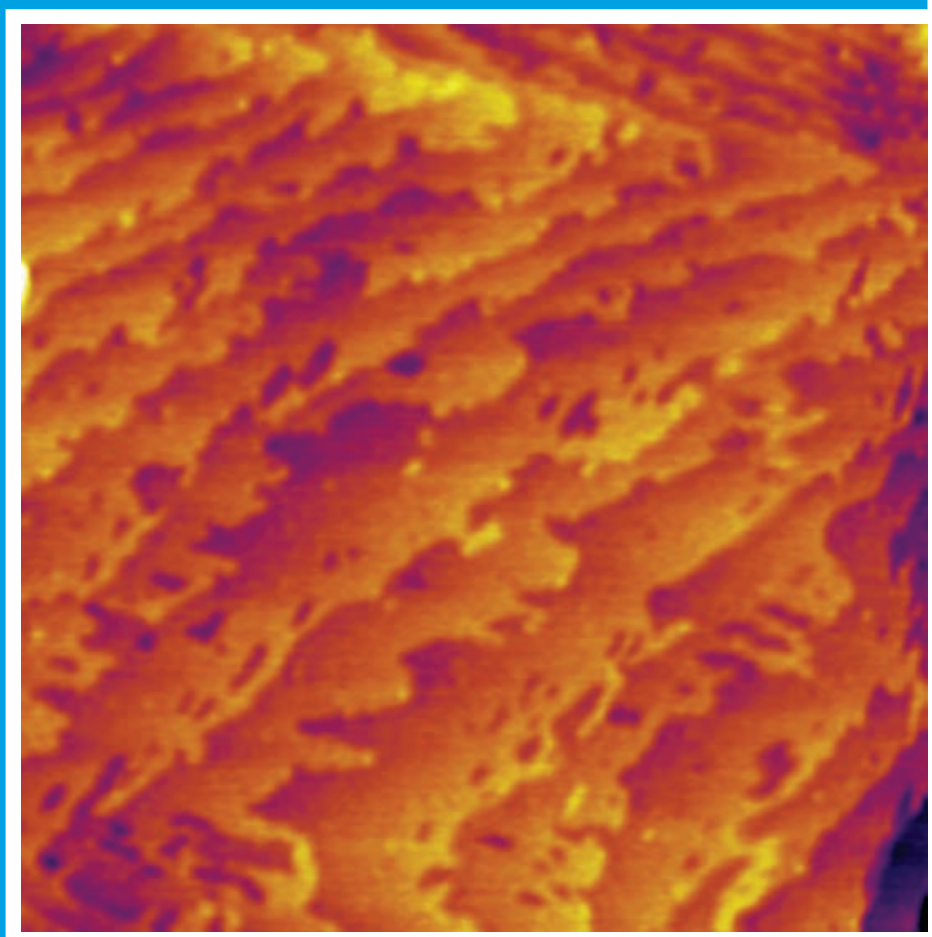

Figure 7: AC-mode closed loop height image of the $\{001\}$ cleavage plane of muscovite mica after etching in hydrofluoric acid for 3 hours. This $512 \times 512$ pixel image was taken using a 10 micron cantilever with a resonance frequency of $4.6 \mathrm{MHz}$ and a response time of $33 \mu \mathrm{s}$. With a line rate of $40 \mathrm{~Hz}$, the image was acquired in less than 13 seconds.

\section{>10X Faster Scanning}

Finally, Cypher supports interchangeable laser spots as small as $3 \mu \mathrm{m}$, allowing the use of small cantilevers (less than 10 microns in length) which, in turn, improve sensitivity and allow extremely high-speed imaging while still maintaining feedback. While fast imaging has been a hot research topic in AFM/SPM recently, most high-speed imaging is done in specialized situations. By far the most common imaging mode AFMs are operated in is AC mode in air (also known as tapping mode, intermittent contact, or dynamic mode). In this mode, the vibrating cantilever itself is the slowest element in the $Z$ feedback loop and it sets the total response speed of the instrument. Current commercial cantilevers with lengths of about 100 microns have response times that are a good fraction of a millisecond. This limits imaging speeds to at most a few lines per second. The small cantilevers enabled by Cypher have response times ten to twenty times faster and allow a commensurate increase in imaging speeds. The image in Figure 7, for example, was generated in just 13 seconds (as compared to many minutes required for older systems) with clearly resolved atomic steps on HF-etched mica.

\section{Other Modes of Operation}

Cypher supports all major scanning and force measurement modes, as well as the new Piezoresponse Force Microscopy (PFM) for high sensitivity, high bias and cross-talk free measurements of piezoelectrics, ferroelectrics and other materials. Cypher also offers built-in nanolithography and nanomanipulation with the highest resolution available.

\section{Come to the Experts.}

Micro-Materia Hitachi High-Te (2) Omnipro

Left Coast Instrumen

XEI Scientific, Inc.

- Represents leading manufacturers

- Supports the Eastern US and Canada

- Offers over 40 years of experience and market knowledge

Need east coast representation?

Have Angstrom Scientific grow your business.

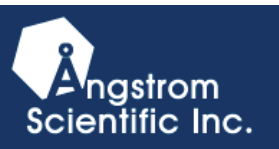

Angstrom Scientific, Inc. (201)-760-2524 Info@angstrom.us www.angstrom.us Imago Millbro

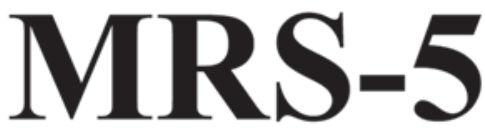

We are ISO-9000 certified and ISO-17025 accredited Microscopy Calibration Standard Now you can calibrate from $1,000 X$ to $1,000,000 X !$

This is our fourth generation, traceable, magnification reference standard for all types (SEM, FESEM, Optical, STM, AFM, etc.) of microscopy. The MRS-5 has multiple $\mathrm{X}$ and $\mathrm{Y}$ pitch patterns ranging from $80 \mathrm{~nm}( \pm 1 \mathrm{~nm})$ to $2 \mu \mathrm{m}$ and $3 \mathrm{bar}$ targets from $80 \mathrm{~nm}$ to $3 \mu \mathrm{m}$. There is also a STM test pattern.

Free web resource guide!
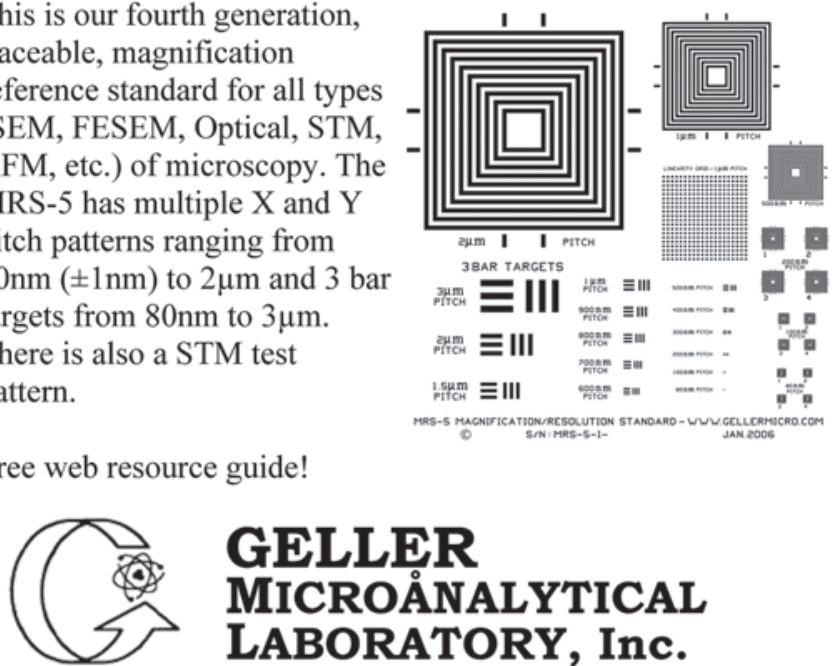

\section{GELLER} MICROÅNALYTICAL LABORATORY, Inc.

426e Boston St., Topsfield, Ma 01983 www.gellermicro.com 